\title{
Coherently delocalized states in dipole interacting Rydberg ensembles: The role of internal degeneracies
}

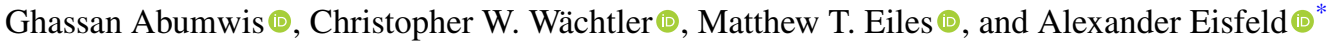 \\ Max-Planck-Institut für Physik komplexer Systeme, Nöthnitzer Strasse 38, D-01187 Dresden, Germany
}

(Received 14 March 2021; accepted 21 June 2021; published 12 July 2021)

\begin{abstract}
We investigate the effect of degenerate atomic states on the exciton delocalization of dipole-dipole interacting Rydberg assemblies. Using a frozen gas and regular one-, two-, and three-dimensional lattice arrangements as examples, we see that degeneracies can enhance the delocalization compared to the situation when there is no degeneracy. This enhancement is particularly large in the case of the three-dimensional (3D) random gas, but is absent for $1 \mathrm{D}$ arrangements. Using the Zeeman splitting provided by a magnetic field, we controllably lift the degeneracy to study in detail the transition between degenerate and nondegenerate regimes. These observations, although specific to the experimentally clean Rydberg gas, have generic implications for various dipole-interacting systems.
\end{abstract}

DOI: 10.1103/PhysRevA.104.013311

\section{INTRODUCTION}

The formation of states where an electronic excitation is coherently delocalized over several particles plays an important role in many systems. Examples include light harvesting in photosynthesis [1], molecular aggregates [2,3], quantum dot arrays [4,5], metallic nanoparticles [6], and Rydberg atoms [7-10]. These coherent collective states are formed by the interaction of transition dipoles of the individual particles. The relevant transition dipoles connect two eigenstates of a particle with different energy, and they are obtained by evaluating the dipole-operator between the respective states. Typically, delocalized states strongly modify the optical properties [3,11] and allow an initially localized excitation to be transferred along the assembly of particles [6,12]. Therefore, many studies of the delocalization properties have been conducted on various ordered or disordered arrangements of interacting particles [13-25].

Although in the majority of cases the involved transitions are nondegenerate, for all of the systems mentioned above there are cases in which transitions within a single particle become degenerate. In the molecular case, such degenerate transition energies can be due to an underlying symmetry [26-30]; similar symmetry-induced degeneracies are present in quantum dots and metal nanoparticles. The spherical symmetry of atoms results in degenerate angular momentum eigenstates. As a result, the dipole-dipole interaction can lead to strong mixing of all degenerate or nearly degenerate many-body states.

\footnotetext{
*eisfeld@pks.mpg.de
}

Published by the American Physical Society under the terms of the Creative Commons Attribution 4.0 International license. Further distribution of this work must maintain attribution to the author(s) and the published article's title, journal citation, and DOI. Open access publication funded by the Max Planck Society.
In this paper, we investigate the effect of such degeneracies on the eigenstate delocalization properties of an assembly of $N$ particles. As a paradigmatic system, we take an assembly of Rydberg atoms. These interact over micron-scale distances due to their large dipole moments, and furthermore these interactions can be tuned across several orders of magnitude by changing the principal quantum number. The relevant interaction between the Rydberg atoms has the same functional form as in molecular systems. Nearly arbitrary arrangements of atoms with relative distances on the order of a few micrometers are possible. The high degree of controllability makes the Rydberg assemblies perfect systems to investigate delocalized excitonic states [7,20,31-36]. The goal of this paper is to examine the effect of energy level degeneracies in a variety of experimentally relevant situations involving Rydberg atoms. This analysis will help to show which of the properties already known from studies of nondegenerate large assemblies cross over when degeneracy is included, reveal ways that the system can be exploited or manipulated due to its inherent degeneracy of states, and give an intuition for how dipolar-interacting systems such as the molecular aggregates or quantum dots mentioned previously are affected by level degeneracy.

For the Rydberg case, the crossover from the degenerate to the nondegenerate system can be studied in a controlled way by applying a static magnetic field $B$. In the strong field limit, the magnetic sublevels are separated by Zeeman splittings exceeding the interaction strengths, which results in energetically well-separated subspaces. Previous studies focused on this situation, finding that many eigenstates are delocalized over a considerable fraction of the Rydberg atoms $[35,36]$. In addition to breaking the energetic degeneracy, application of a strong magnetic field also leads to a typically nonisotropic interaction with a specific angular dependence, which resembles that of aligned dipoles in the direction of the magnetic field. It was found that this nonisotropic interaction leads to very different delocalization properties compared to an isotropic interaction [35]. In particular, the delocalization is much larger in the anisotropic case than in the isotropic one, 
raising the question of how delocalization occurs in the fieldfree case where the atoms no longer have a preferred direction.

We find that the delocalization is enhanced by the degeneracies at zero field, resulting in even larger delocalization lengths. Surprisingly, these grow even further at small but nonzero magnetic fields, before reducing to the $B \rightarrow \infty$ limit studied in Refs. [35,36] at higher fields. To better understand the origin of these observations, we use one-, two-, and three-dimensional lattice arrangements of the atoms [37-41] to systematically probe delocalization in systems ranging from the case we previously studied-corresponding to a disordered three-dimensional lattice with fractional filling of atoms- to fully structured or low-dimensional systems. By applying also a magnetic field as we transition from regular to irregular atomic positions, we can obtain further insight into the delocalization properties of this system. These findings can help to design and interpret Rydberg experiments where large delocalization is desired, and they have implications for the behavior of the general class of dipole-dipole interacting systems mentioned above.

\section{INTERACTING RYDBERG ATOMS}

The role of atomic degeneracies in delocalization can be clearly studied using the spin-independent Rydberg states $|v, l, m\rangle$, where $v$ denotes the principal quantum number, $l$ is the orbital angular momentum, and $m$ is the corresponding magnetic quantum number. The simplest case involves interacting $s$ - and $p$-states, i.e., $l=0$ and 1 , with the same $v$. Without loss of generality, we choose the $m$ quantization axis to be the same for all atoms. There are two manifolds of states for each atom:

$$
\begin{gathered}
|\uparrow, m\rangle \leftrightarrow|p, m\rangle \quad \text { with } m=0, \pm 1, \\
|\downarrow, m\rangle \leftrightarrow|s, m\rangle \quad \text { with } m=0 .
\end{gathered}
$$

We set the energy of the $s$-state, which does not depend on magnetic field strength, to be the reference (zero) energy. The $p$-state energies depend linearly on an applied magnetic field via the Zeeman shift,

$$
\epsilon_{m}(B)=\epsilon+\mu_{\mathrm{B}} m B,
$$

where $\epsilon$ is the energy difference between the field-free $p$ - and $s$-states. The level structure of our effectively two-level system is shown in Figs. 1(a) and 1(b).

We consider an interacting system of $N$ of these two-level atoms, described by the Hamiltonian

$$
H=\sum_{\alpha=1}^{N} H^{(\alpha)}+\sum_{\alpha=1}^{N} \sum_{\beta<\alpha} V^{(\alpha, \beta)},
$$

where $H^{(\alpha)}$ denotes the Hamiltonian of particle $\alpha$, and $V^{(\alpha, \beta)}$ is the dipole-dipole interaction between the atoms,

$$
V^{(\alpha, \beta)}=\frac{\vec{\mu}_{\alpha} \cdot \vec{\mu}_{\beta}}{R_{\alpha, \beta}^{3}}-3 \frac{\left(\vec{\mu}_{\alpha} \cdot \vec{R}_{\alpha, \beta}\right)\left(\vec{\mu}_{\beta} \cdot \vec{R}_{\alpha, \beta}\right)}{R_{\alpha, \beta}^{5}} .
$$

Here, $\vec{R}_{\alpha, \beta}$ is the distance vector between the two particles, and $R_{\alpha, \beta}$ denotes its magnitude. The dipole operator of atom $\alpha$ is denoted by $\vec{\mu}_{\alpha}$.

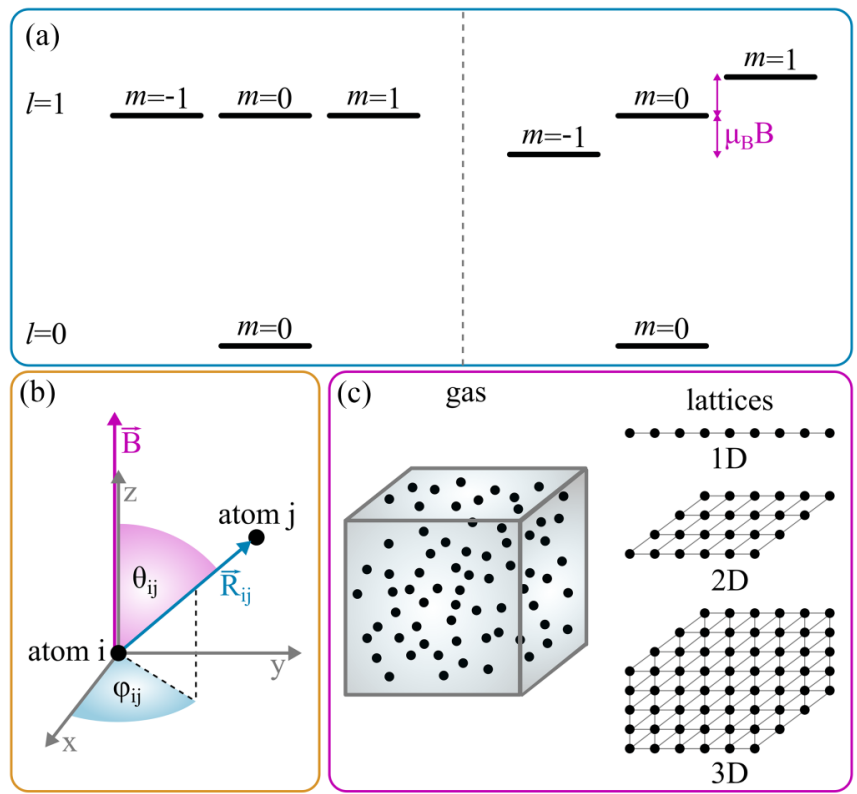

FIG. 1. (a) Energy structure of a single Rydberg atom in the relevant subspace without (left) and with (right) an applied magnetic field. The $l=0, m=0$ levels are set to be at zero energy while the $l=1, m=0$ state sits at energy $\epsilon$. (b) Definition of the angles that enter the interaction matrix elements. The angle $\theta_{i j}$ is defined with respect to the quantization axis, while the angle $\phi_{i j}$ is given with respect to an arbitrarily chosen $x$-axis. When a magnetic field is present, we choose the quantization axis $\vec{z} \| \vec{B}$. (c) We structure our study around four generic arrangements: a three-dimensional random gas, and one-, two-, and three-dimensional regular lattice arrangements.

We are interested in the situation when there is one excitation in the system. Consequently, we choose basis states with one atom excited to the $p$-state and the remaining atoms still in the $s$-state. We denote these states as

$$
\left|j, m_{j}\right\rangle \equiv|s, 0\rangle \cdots\left|p, m_{j}\right\rangle \cdots|s, 0\rangle,
$$

where $j$ identifies the atom that is excited to the $p$-state. The matrix elements of the Hamiltonian (4) in this basis are then given by

$$
\left\langle j, m_{j}|H| i, m_{i}\right\rangle=\delta_{j i} \delta_{m_{j} m_{i}} \epsilon_{m_{j}}(B)+\frac{\mu_{s p}^{2}}{R_{j i}^{3}} M_{j, i}^{m_{j}, m_{i}} .
$$

Here we have introduced the transition dipole moment $\mu_{\mathrm{sp}}=$ $\langle v, l=0|r| v, l=1\rangle$ and a matrix element encoding the relative orientation of the atoms with respect to one another and with respect to the quantization axis,

$$
\begin{gathered}
M_{i, j}^{0,0}=\frac{1-3 \cos ^{2} \theta_{i j}}{3}, \\
M_{i, j}^{+1,+1}=M_{i, j}^{-1,-1}=-\frac{M_{i, j}^{0,0}}{2}, \\
M_{i, j}^{-1,0}=\frac{e^{-i \phi_{i j}}}{\sqrt{2}} \cos \theta_{i j} \sin \theta_{i j}, \\
M_{i, j}^{+1,0}=-M_{i, j}^{-1,0}, \\
M_{i, j}^{-1,+1}=\frac{e^{-2 i \phi_{i j}} \sin ^{2} \theta_{i j}}{2} .
\end{gathered}
$$



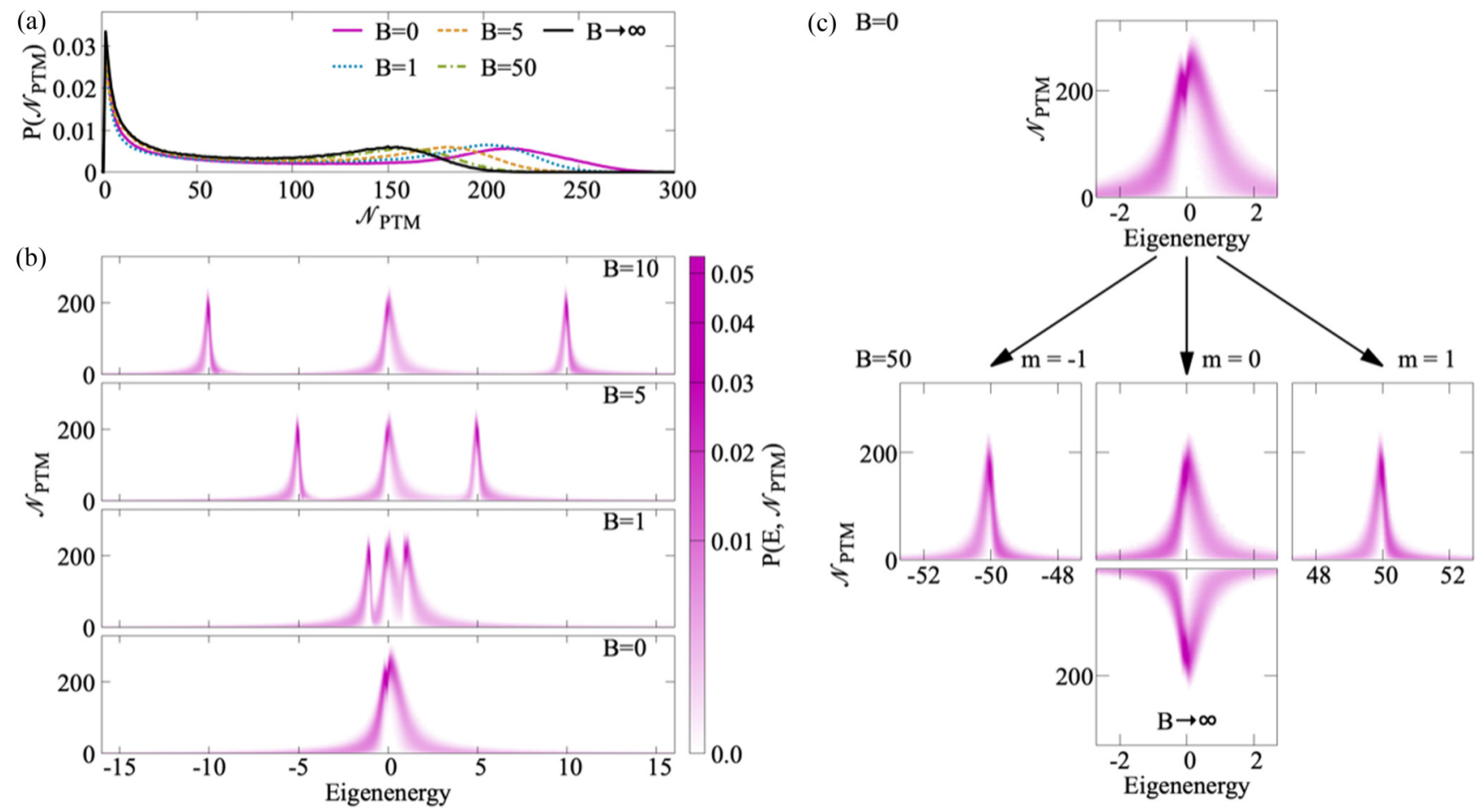

FIG. 2. The frozen Rydberg gas. All plots are for $N=1000$ Rydberg atoms averaged over $\sim 10^{3}$ realizations. (a) Probability densities for the PTM measure, displayed for several magnetic field strengths. The black curve gives the $B \rightarrow \infty$ reference value, obtained by setting the coupling between $m$-levels to zero. (b) Probability densities to find a certain PTM value for an eigenstate with a certain energy, for the same magnetic fields as in panel (a). (c) Comparison between the $B=0$ and 50 case. Although we use $m$ as a label to distinguish the three distributions, we note that they are calculated in the same way for each magnetic field value, and these labels are only approximate, since the Zeeman splitting allows these distributions to be characterized by $m$ to a very good approximation. Below the $m=0$ distribution, we show the distribution in the asymptotic limit $B \rightarrow \infty$ (flipped to better see the close agreement with the $m=0$ distribution for $B=50$ ). The energies are given in units of $E_{\mathrm{ref}}=\mu_{\mathrm{sp}}^{2} / 3 a_{\mathrm{ref}}^{3}$, where $a_{\mathrm{ref}}=(3 / 4 \pi N)^{1 / 3} L$ is the Wigner-Seitz radius. The zero of energy is at the energy $\epsilon$ of the noninteracting atoms, which is introduced in Eq. (3).

Interchanging indices results in complex conjugation. The angle between the quantization axis and the distance vector $\vec{R}_{i j}$ is $\theta_{i j}$, and $\phi_{i j}$ is the azimuthal angle between the $x$-axis and the projection of the distance vector onto the $x-y$ plane [see Fig. 1(b)].

In the limit where the magnetic field is much larger than the coupling strength $M_{i j}^{m m^{\prime}}$, with $m \neq m^{\prime}$, the Hamiltonian effectively decomposes into three subspaces, one for each magnetic quantum number $m$. In each subspace, the interaction between atoms is given by $M_{i j}^{m m}$, whose angular dependence is given by $1-3 \cos ^{2} \theta_{i j}$. This is the anisotropic interaction studied in $[35,36]$. This function can evaluate to both positive and negative values and becomes zero at the so-called "magic angle." We note that the coupling between the different $m$-levels has a completely different angular dependence. In particular, at the magic angle there is now a strong coupling between different $m$-levels, so that reducing the magnetic field from the strong-field limit reduces the relevance of the magic angle.

\section{EIGENSTATES AND DELOCALIZATION MEASURE}

The eigenstates $\left|\psi_{\ell}\right\rangle$ and eigenenergies $E_{\ell}$ follow from the time-independent Schrödinger equation

$$
H\left|\psi_{\ell}\right\rangle=E_{\ell}\left|\psi_{\ell}\right\rangle
$$

In the basis (6), the eigenstates can be written as

$$
\left|\psi_{\ell}\right\rangle=\sum_{j} \sum_{m_{j}} c_{j, m_{j}}^{(\ell)}\left|j, m_{j}\right\rangle
$$

The absolute square of the coefficients $c_{j, m_{j}}^{(\ell)}$ is the probability to find the excitation on particle $j$ in the specific state $\left|\uparrow, m_{j}\right\rangle$. We obtain the eigenenergies $E_{\ell}$ and the eigenstate coefficients $c_{j, m_{j}}^{(\ell)}$ [cf. Eqs. (14) and (6)] by diagonalization of a matrix with matrix elements given by $\left\langle j, m_{j}|H| i, m_{i}\right\rangle$ of Eq. (7).

We are interested in the overall delocalization of the excitation, roughly corresponding to the number of atoms that participate in a given eigenstate. This is given by the probability that a particle is in the $\uparrow$-manifold. Since the decomposition into individual $m$ levels is irrelevant to the overall excitation delocalization, we sum over these levels to obtain the probability that the excitation is on particle $j$,

$$
P_{j}^{(\ell)}=\sum_{m_{j}}\left|c_{j, m_{j}}^{(\ell)}\right|^{2} .
$$

A convenient measure of delocalization can be obtained by counting the number of atoms involved in a state $\ell$ that have an excitation probability larger than a chosen threshold $P_{\text {thresh }}$. We will refer to this as the "population threshold measure" 
(PTM),

$$
\mathcal{N}_{\mathrm{PTM}}^{(\ell)}=\sum_{j} \Theta\left(P_{j}^{(\ell)}-P_{\text {thresh }}\right),
$$

where $\Theta$ denotes the Heaviside step function, i.e., $\Theta(P)=$ 1 for $P \geqslant 0$ and $\Theta(P)=0$ for $P<0$. We use $P_{\text {thresh }}=1 / N$; this gives a PTM limit of $N$ in a fully delocalized, equally distributed state, and 1 for a state localized on a given atom.

In our previous works $[35,36]$ we used the so-called "coherence" measure to quantify the delocalization of the excitation. Since the PTM measure works directly with the populations, it is more suitable for the present situation where we are not interested in the coherence properties of the reduced density matrix. In the supplemental material of Ref. [36] we compared these measures for the case without $m$ levels, and we observed that they are essentially proportional.

\section{THE FROZEN RYDBERG GAS}

In a frozen Rydberg gas, the atoms are randomly distributed within a certain volume and, due to the typical ultracold laboratory conditions and relevant timescales, remain motionless during the course of excitation and measurement of delocalized states. For a representative study we consider $N=1000$ Rydberg atoms with random positions placed uniformly inside a cubic volume with length $L$; the results are nearly independent of boundary conditions $[35,36]$. We have also studied the system's dependence on the number of atoms $N$, finding that the PTM distribution is largely composed of a peak at low PTM values which is independent of $N$ and a peak at large PTM values, whose position and width scale linearly with $N$. This was previously observed for the large magnetic field case [35]. It is convenient to use the Wigner-Seitz radius $a_{\text {ref }}=(3 / 4 \pi N)^{1 / 3} L$ as the unit of distance, and for the unit of energy we use $E_{\mathrm{ref}}=\mu_{s p}^{2} / 3 a_{\mathrm{ref}}^{3}$. From Eq. (7) we see that the density simply scales the strength of the interaction, which is reflected by our choice of the energy unit. Therefore, a change in density will not change the results presented below. We average over $10^{3}$ independent random gas realizations. For each realization we obtain the eigenenergies $E_{\ell}$ and corresponding PTM value $\mathcal{N}_{\mathrm{PTM}}^{(\ell)}$ for all $3 \cdot N$ eigenstates. In the following, we focus on the probability $P\left(E, \mathcal{N}_{\text {PTM }}\right)$ to obtain a certain $\mathcal{N}_{\text {PTM }}$ at the energy $E$. These distributions are obtained by binning the $E_{\ell}, \mathcal{N}_{\mathrm{PTM}}^{(\ell)}$ pairs to a regularly spaced grid. By integrating over energy, we can also obtain the marginal distribution $P\left(\mathcal{N}_{\text {PTM }}\right)$.

In Fig. 2 the dependence of $P\left(E, \mathcal{N}_{\text {PTM }}\right)$ and $P\left(\mathcal{N}_{\text {PTM }}\right)$ on the magnetic field strength is shown. Figure 2(a) shows the probability density $P\left(\mathcal{N}_{\mathrm{PTM}}\right)$ for several magnetic field strengths. For all magnetic field strengths there is a large fraction of states with PTM on the order of 150-200, i.e., the delocalization is spread over nearly $20 \%$ of the atoms in the gas. The PTM distribution is shifted toward larger values for all finite $B$-fields in comparison to the $B \rightarrow \infty$ case, to which they converge. The peak at low PTM stems from clusters-dimers, trimers, etc.-formed from strongly interacting atoms in relatively close proximity. These cluster states decouple from the system, leaving a residual gas with more homogeneous interparticle interactions, which in turn lead to large extended states [36].

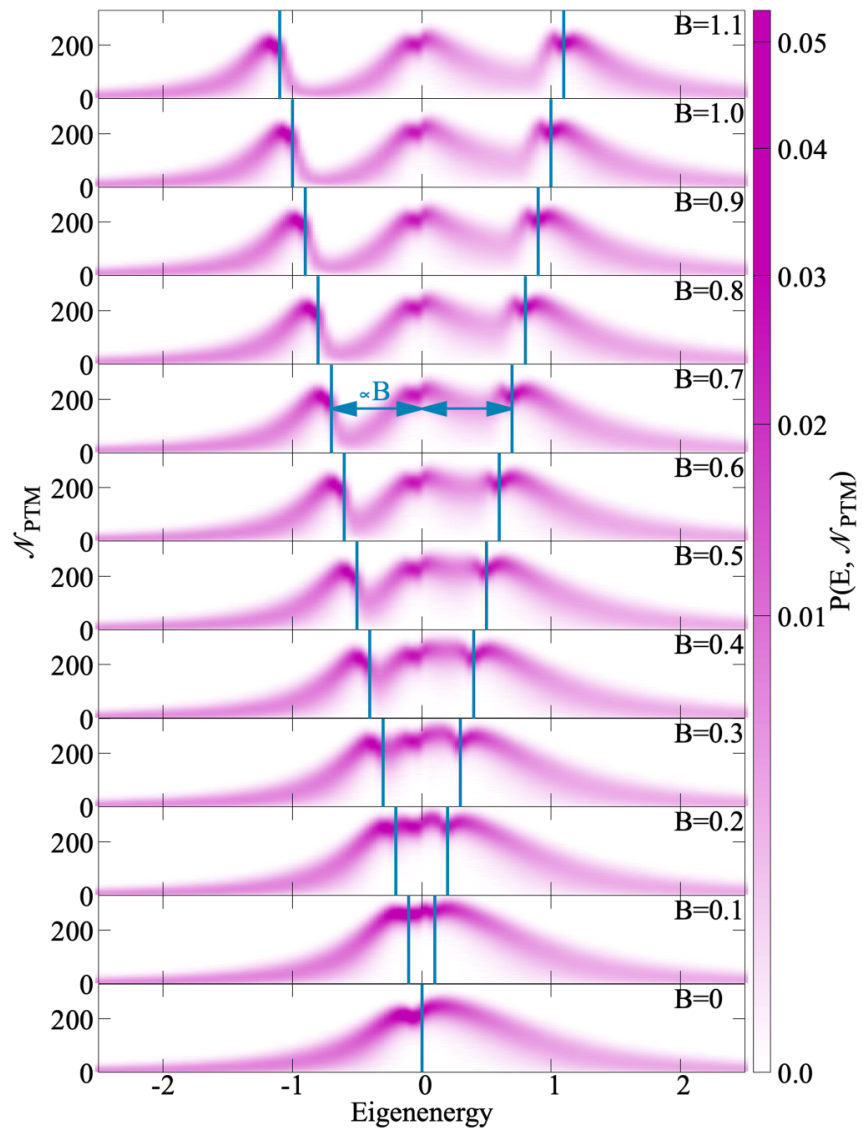

FIG. 3. Same as Fig. 2(b), but for small magnetic fields. The vertical bars indicate the positions of the Zeeman energies, $\pm m B$.

This interpretation is supported by the energy-resolved PTM distributions shown in Fig. 2(b). These reveal immediately that the delocalized states cluster around zero energy while the cluster states with low PTM are primarily found in the wings of this distribution. One sees additional structure in the PTM distributions: at $B=0$ the distribution has an asymmetric double-peak structure and is even broader than in the $B \rightarrow \infty$ case. For intermediate $B$ the distribution splits into three peaks whose centers follow the Zeeman energies proportional to the magnetic field strength $B$. For $B \gtrsim 10$ the three well-separated peaks each have states with only a single $m$ value. These peaks have a similar shape, but different width and "orientation." While $B=10$ is not quite sufficient to reach the asymptotic $B \rightarrow \infty$ distribution, $B=50$, shown in panel (c), is. One clearly sees that the $m= \pm 1$ peaks are mirror images of the $m=0$ peak with half the width, features that result from the form of the interaction matrix. In the $B \rightarrow \infty$ limit, the off-diagonal couplings in $M$ can be ignored, and thus the Hamiltonian separates into three blocks, with energies $m B$ on the diagonal and off-diagonal elements given by $M_{i, j}^{0,0}$, $M_{i, j}^{-1,-1}$, and $M_{i, j}^{+1,+1}$. From Eqs. (8)-(12) one sees that $M_{i, j}^{-1,-1}$ and $M_{i, j}^{+1,+1}$ have the same sign and magnitude, but a different sign and half the magnitude of the $M_{i, j}^{0,0}$ interaction. It is clear that the PTM distributions for all three blocks are identical up to a scale factor, since the interactions are proportional. Mirrored below the $m=0$ distribution, we show the asymptotic 

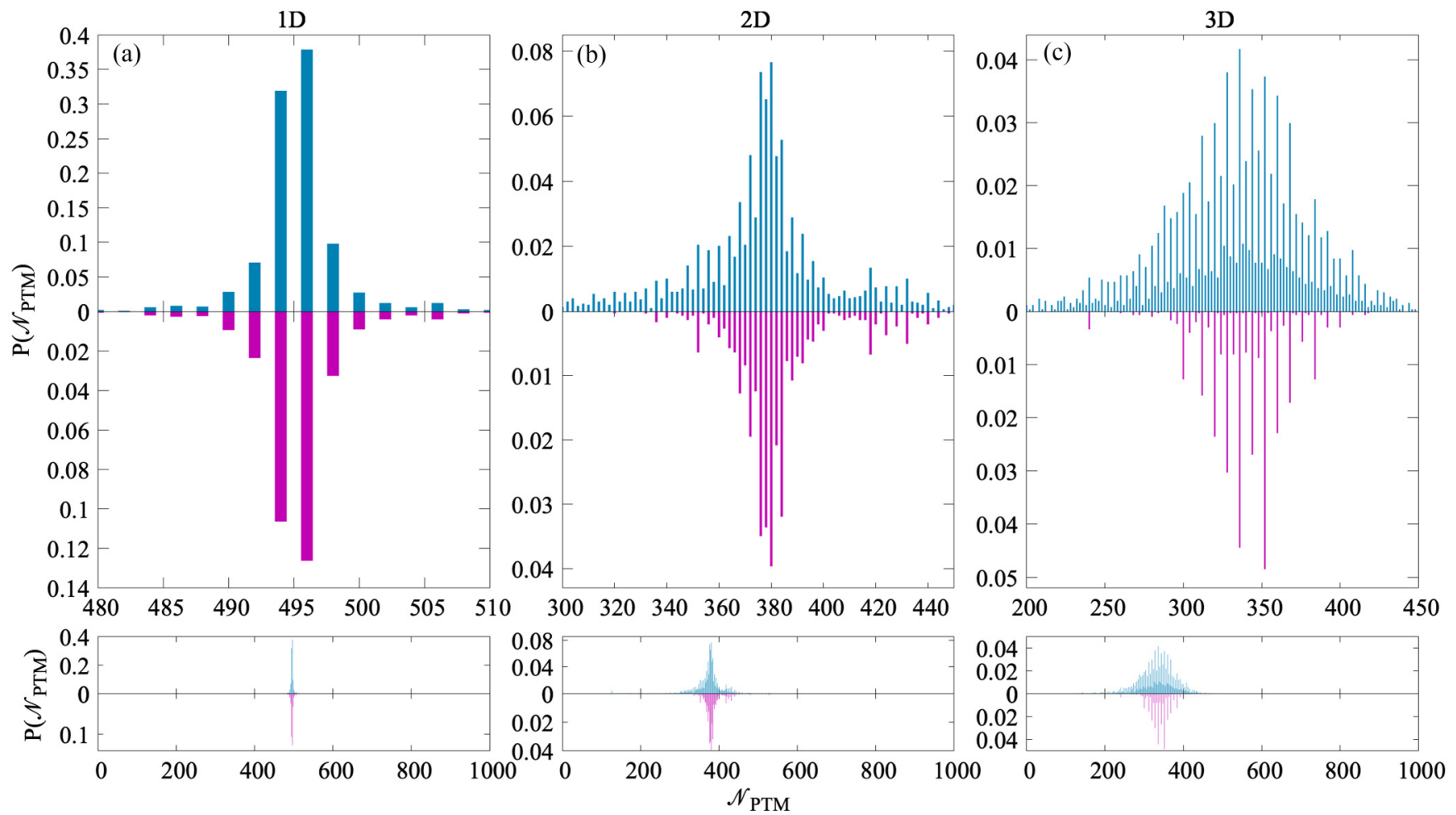

FIG. 4. Distribution of PTM for (a) a 1D chain, (b) a 2D, and (c) a 3D lattice. In all three cases we have roughly the same number of atoms as in the frozen gas case [990 atoms in the 1D case $\left(N_{x}=990\right), 992$ atoms in the $2 \mathrm{D}$ case $\left(N_{x}=32, N_{y}=31\right)$, and 990 atoms in the 3D case $\left.\left(N_{x}=11, N_{y}=10, N_{z}=9\right)\right]$, and the quantization axis is set parallel to the magnetic field, pointing along the $z$-direction. The blue bars are the case without magnetic field $(B=0)$, and the magenta (mirrored) bars are the infinite B-field limit. The bottom row shows the upper plots on the same $x$-axis.

$B \rightarrow \infty$ result [36]. It is identical to the middle peak of the $B=50$ distribution, confirming the validity of the results of Ref. [36], since the nondegenerate regime is reached in the limit of moderately high magnetic fields. We can therefore identify the three distributions that appear at large magnetic fields with $m$ labels. Specifically, at typical Rydberg densities the interaction strength is on the order of a few $\mathrm{MHz}$, which requires a magnetic field on the order of $10 \mathrm{G}$ to reach the separated $m$-level regime.

To study in more detail the splitting of the asymmetric $B=0$ distribution into three peaks, in Fig. 3 we focus on the region $B \leqslant 1.1$. Surprisingly, the apparently monotonic decrease in delocalization extent with increasing $B$ seen in Fig. 2 does not hold all the way to $B=0$, and in fact the largest delocalized states are seen for small but nonzero fields with partially lifted degeneracy. For $B \lesssim 1$ the magnetic field is too weak to separate the three distributions fully, resulting in a complicated distribution with several maxima culminating in a clearly emerging triple-peak structure as $B$ grows to $\approx 1$. The peak centered at zero detuning has a similarly asymmetric form as it does when $B=0$, but with a smaller width and reduced maximum value. The right peak $(m=$ +1 ) has a strongly asymmetric shape and exhibits a similar double-peak structure as the $m=0$ peak. In contrast, the left peak $(m=-1)$ has no double-peak structure and its asymmetry is mirrored with respect to the $m=+1$ peak. Recall that in the large $B$-field case, these two peaks are identical.
From these calculations we see that the degenerate sublevels increase the extent of delocalization and add additional structure to the random Rydberg gas. Turning on a magnetic field leads to complicated behavior, even including an increase in the delocalization length at small magnetic fields and hence a small lifting of the degeneracy, which eventually converges to the nondegenerate $B \rightarrow \infty$ case studied in Refs. [35,36].

To better understand the effect of the degenerate atomic transitions, we now consider different lattice arrangements.

\section{ATOMS ARRANGED ON A LATTICE}

\section{A. One-dimensional chain}

We consider $N$ equidistant atoms placed in a onedimensional (1D) lattice. We first note that the angle between the quantization axis and the vector $\vec{R}_{i j}$ is the same for all pairs of atoms, i.e., $\theta_{i j} \equiv \theta$ and $\phi_{i j} \equiv \phi$ for all $i$ and $j$. The Hamiltonian can therefore be simplified to

$$
H=\sum_{i, j} \sum_{m, m^{\prime}}\left[\epsilon_{m}(B) \delta_{m, m^{\prime}} \delta_{i j}+\frac{\mu_{\mathrm{sp}}^{2}}{R_{i j}^{3}} M_{m, m^{\prime}}\right]|i, m\rangle\left\langle j, m^{\prime}\right|,
$$

where $M^{m, m^{\prime}}=M_{i, j}^{m, m^{\prime}}$ are independent of atom indices. This has far-reaching consequences. As shown in Appendix A, the PTM distribution is actually independent of the direction and strength of the magnetic field, in pronounced contrast to the three-dimensional gas, and even though the eigenvalues depend on the magnetic field's strength and direction. This 
holds even when the atoms are not placed equidistantly. The PTM for $N=990$ is shown in Fig. 4(a) for $B=0$ and $B \rightarrow \infty$ (mirrored below). The PTM distribution is centered around $N / 2$, in excellent agreement with the analytic estimates discussed in Appendix B.

\section{B. Two-dimensional lattice}

We now place the atoms in a two-dimensional (2D) rectangular lattice in a plane perpendicular to the quantization axis so that $\theta_{i j}=\pi / 2$ for all $i, j$. In this case $M_{i, j}^{ \pm 1,0}=M_{i, j}^{0, \pm 1}=0$, and thus the $m=0$ subspace decouples from the $m= \pm 1$ states. Within the $m=0$ subspace the interaction $\mu_{\mathrm{sp}}^{2} / 3 R_{i j}^{3}$ is isotropic, and was previously studied without the lattice arrangement in Ref. [35]. The $m=0$ PTM distribution is independent of $B$. In Fig. 4(b) we contrast the full PTM distribution for the case $B=0$ (top panel) with the $B \rightarrow \infty$ case (bottom panel), with the magnetic field perpendicular to the lattice. The two distributions are not equal due to the $m= \pm 1$ states present only in the $B=0$ case. By comparing the differences between the two mirrored distributions, it is apparent that these states both increase the number of highly delocalized states and give rise to several somewhat more localized states, which are completely absent in the $B \rightarrow \infty$ case. We notice that the increase in the number of delocalized states is rather small, and in particular the maximal value of $\mathcal{N}_{\text {PTM }}$ remains essentially unchanged. We found the same behavior, which is in sharp contrast to the 3D case, also for a random 2D gas. Unlike in the $1 \mathrm{D}$ case, in the 2D case with $B \neq 0$ both the eigenstates and the eigenenergies depend on the magnetic field orientation. We note that the Hamiltonian resulting from the $B$-field orientation discussed here has the same general form as those discussed in the context of topological band structure in dipole-interacting systems [30,42].

\section{Three-dimensional lattice}

A three-dimensional (3D) lattice bears the closest resemblance to the frozen gas. Figure 4(c) shows that the coupling between $m$ levels still has only a small impact on the delocalization, which, as in the $1 \mathrm{D}$ and $2 \mathrm{D}$ cases, is characterized by PTM values around $N / 2$, although in $3 \mathrm{D}$ the distribution is broader. In marked contrast to the $1 \mathrm{D}$ and $2 \mathrm{D}$ cases, there are now states with larger PTM values than in the infinite $B$-field limit. We therefore see that the main finding of the previous section, namely that the degenerate sublevels at $B=0$ lead to larger delocalization than in the nondegenerate $B \rightarrow \infty$ limit, only occurs for the 3D arrangement.

There are several differences between the 3D-lattice and the random gas PTM distributions. Most notably, the lattice PTM distribution consists only of a single-peaked and relatively narrow distribution centered at a high PTM value of approximately $N / 3$; in contrast, the random gas case exhibits a very broad distribution with two major peaks at PTM values around $\mathcal{N}_{\text {PTM }}=2$ and at $\mathcal{N}_{\text {PTM }} \approx N / 5$. These differences are explained by the absence of strongly interacting clusters in the lattice.

To study the transition from the 3D lattice case to the frozen gas, we introduce now a lattice that is only partially filled with atoms, characterized by the filling fraction $f$. For a given $f$, we adjust the size of the lattice such that we

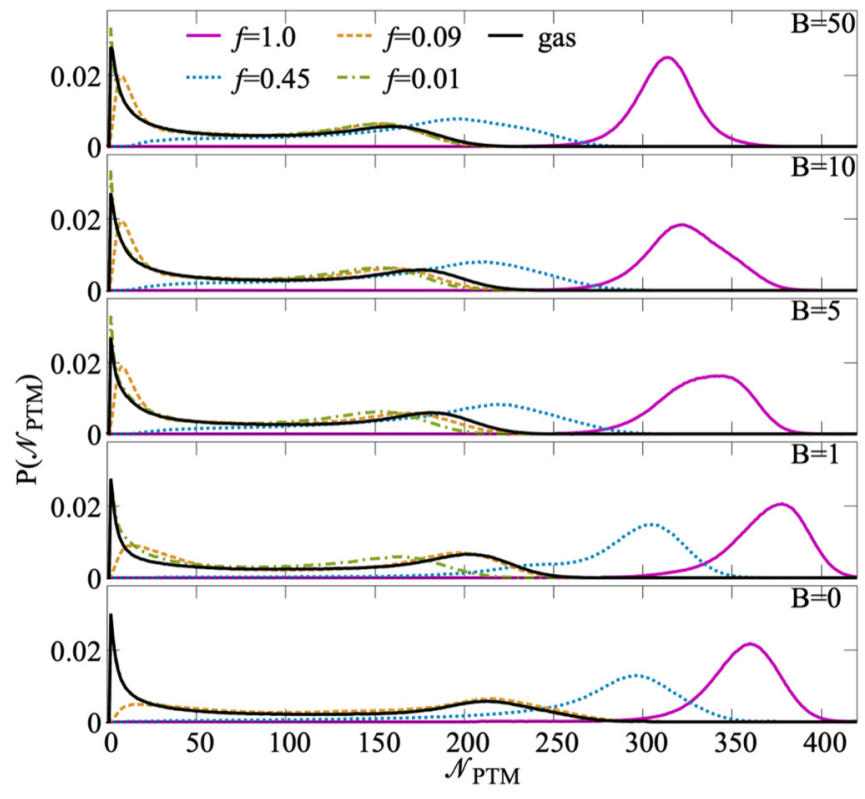

FIG. 5. Transition from lattice to gas. Comparison of a 3D lattice with different filling fractions (legend provided in the upper panel) to the frozen Rydberg gas (black) for different strengths of the magnetic field (provided in the panels). For all cases we fix the number of atoms at $N=990$. For the lattice we apply 5\% uniform fluctuations around the perfect lattice positions.

always have the same total number of atoms in the system. This introduces clustering effects into the arrangement of the atoms. In Fig. 5 we show the PTM distribution for several filling fractions and magnetic fields. To smoothen the distribution with a high filling fraction, we added a small (5\% of the lattice constant) disorder in the position of the atoms around the lattice positions. As expected, the peak in the PTM distribution starts to broaden and shifts to smaller values as $f$ decreases. For $f=0.09$, a peak at small $(<50)$ PTM values develops, becoming more pronounced at higher $B$ values. We note that for $B=0$ and for large magnetic field $(B=50)$, the distribution at small filling fraction $(f=0.01)$ agrees nearly perfectly with the frozen gas distribution (shown as a black line). Curiously, at intermediate magnetic field strengths the distributions with small filling fractions have peaks at smaller PTM values than the frozen gas case.

\section{DETERMINATION OF THE DELOCALIZATION}

As discussed in Refs. [35,36], one way to access the eigenstates $\left|\psi_{\ell}\right\rangle$ is via microwave transitions from an initial state with all atoms prepared in the $s$-state. By scanning the microwave frequency, one can select states at different eigenenergies $E_{\ell}$. In the lattice case discussed above, where the atoms are deterministically placed, one could then also determine the $p$-population of each atom, as done in Refs. [12,31]. From the definition of (15) we see that the populations fully determine the PTM, thus directly giving access to the delocalization of the eigenstates. The absorption strength for the transition from the initial state $|s \cdots s\rangle$ to the final state $\left|\psi_{\ell}\right\rangle$, for a microwave with polarization $\varepsilon$ and resonant with the state's transition 
frequency, is determined by $\left|\left\langle s \cdots s\left|\sum_{\alpha} \vec{\mu}_{\alpha} \cdot \vec{\varepsilon}\right| \psi_{\ell}\right\rangle\right|^{2}=$ $\left|\sum_{\alpha} \sum_{m_{\alpha}}\left(\vec{\mu}_{\mathrm{sp}} \cdot \vec{\varepsilon}\right) c_{\alpha, m_{\alpha}}^{(\ell)}\right|^{2}$. In general, the eigenstates have very different absorption strengths, and for many arrangements there are several eigenstates that have vanishingly small transition strengths.

Another possibility to probe the eigenstates in the lattice case is via the excitation scheme employed in recent experiments of the Ott group [43]. First, we prepare all atoms but one in the $s$-state, and we denote the atom which is still in the ground state $g$ by $\alpha_{0}$. Via laser excitation one can again address the eigenstate $\left|\psi_{\ell}\right\rangle$, but now with transition probability $\left|\left\langle s \cdots g_{\alpha_{0}} \cdots s\left|\sum_{\alpha} \vec{\mu}_{\alpha} \cdot \vec{\varepsilon}\right| \psi_{\ell}\right\rangle\right|^{2}=$ $\left|\sum_{m_{\alpha_{0}}}\left(\vec{\mu}_{g p}^{m_{\alpha}} \cdot \vec{\varepsilon}\right) c_{\alpha_{0}, m_{\alpha_{0}}}^{(\ell)}\right|^{2}$. The excitation probability in this scenario is determined by the eigenstate coefficient $c_{\alpha_{0}, m_{\alpha_{0}}}^{(\ell)}$ that corresponds to the atom that was initially prepared in the ground state. For both the microwave and the laser excitation scheme, the transition probabilities depend on the polarization $\varepsilon$ of the microwave or laser, respectively. We believe that with the laser excitation scheme one can indeed access most, if not all, eigenstates. The p-population of each atom can be determined for the case of the laser excitation in the same way as described above for the case of microwave excitation. Finally, we remark that one can also use transfer experiments to obtain information about the eigenstates [20].

\section{CONCLUSIONS}

In this paper, we have explored the influence of degenerate sublevels on the extent of single exciton state delocalization in a Rydberg atom assembly. For degenerate sublevels, we find in the three-dimensional system larger delocalization than for the separate (nondegenerate) $m$-level manifolds. In one and two dimensions, the inclusion of $m$-level degeneracies does not lead to much larger delocalization in comparison with the nondegenerate case. By applying a homogeneous magnetic field, one can not only split the $m$-levels of a single atom, but also induce a preferred orientation and, with that, the angular dependence of the interaction. In one dimension we find that the magnetic field orientation does not influence the delocalization properties of the system at all, and only weakly in three dimensions. The situation in two dimensions is very different. For large magnetic fields, the direction of the field determines whether the interaction is predominately isotropic or anisotropic. For magnetic fields perpendicular to the plane, the interaction is isotropic, whereas the interaction has the angle dependence of parallel aligned dipoles, where, for example, the "magic angle" plays an important role, when the fields are parallel to the plane. This situation mimics the situation of regular 2D molecular arrangements on surfaces. For example, for PTCDA-molecules on a $\mathrm{KCl}$ surface, all molecular transition dipoles are oriented along the diagonal of the lattice [44,45], an interesting situation that can be simulated in the Rydberg assembly. In the present work, we focused on the case of a magnetic field that is perpendicular to the plane of the Rydberg atoms. For this situation, we did not find a large variation of the delocalization as a function of the magnetic field strength, but we do still see the general trend that the delocalization decreases with increasing magnetic field.

Our choice of $s$ and $p$ states to study exciton delocalization was made in order to introduce a tractable level of degeneracy to the system. If instead we had chosen $p$ and $d$ states as $\downarrow$ and $\uparrow$ states, respectively, then in addition to a small increase in the degeneracy of $\uparrow$ states, we would introduce doubly degenerate $\downarrow$ states, leading to an exponential growth in the number of aggregate basis-states. Such a case is challenging to treat numerically, but is the typical situation in molecular systems. Inclusion of Rydberg fine structure also leads to such a scenario for all possible states, since the spin degree of freedom leads even to a doubly degenerate $s$ state. Exploration of this physics would be theoretically interesting along the lines of choosing other degenerate states, but is also necessary in order to treat realistic experimental conditions $[43,46]$.

\section{ACKNOWLEDGMENTS}

We acknowledge funding from the Deutsche Forschungsgemeinschaft (DFG): Grant No. EI 872/4-1 through the Priority Programme SPP 1929 (GiRyd). A.E. acknowledges support from the DFG via a Heisenberg fellowship (Grant No. EI 872/5-1). M.T.E. acknowledges support during the early stages of the research from a postdoctoral fellowship from the Alexander von Humboldt Stiftung. C.W.W. acknowledges support from the Max-Planck Gesellschaft via the MPI-PKS Next Step fellowship.

\section{APPENDIX A: EIGENSTATE STRUCTURE IN THE 1D CHAIN}

The Hamiltonian of a 1D chain, Eq. (17), can be written in matrix form as

$$
H(B)=\underline{\epsilon(B)} \otimes \underline{I}+\underline{M} \otimes \underline{\underline{V}} .
$$

Here a single underbar denotes a $3 \times 3$ matrix, and a double underbar denotes an $N \times N$ matrix. The symbol $\otimes$ denotes the Kronecker product between matrices. These matrices are given by

$$
\underline{\epsilon(B)}=\left(\begin{array}{ccc}
-\mu_{\mathrm{B}} B & 0 & 0 \\
0 & 0 & 0 \\
0 & 0 & +\mu_{\mathrm{B}} B
\end{array}\right)
$$

and

$$
\underline{M}=\left(\begin{array}{ccc}
\frac{3 \cos ^{2} \theta-1}{6} & \frac{e^{-i \phi}}{\sqrt{2}} \cos \theta \sin \theta & \frac{e^{-2 i \phi} \sin ^{2} \theta}{2} \\
\frac{e^{i \phi}}{\sqrt{2}} \cos \theta \sin \theta & \frac{1-3 \cos ^{2} \theta}{3} & -\frac{e^{-i \phi}}{\sqrt{2}} \cos \theta \sin \theta \\
\frac{e^{2 i \phi} \sin ^{2} \theta}{2} & -\frac{e^{i \phi}}{\sqrt{2}} \cos \theta \sin \theta & \frac{3 \cos ^{2} \theta-1}{6}
\end{array}\right)
$$


The matrix $\underline{\underline{V}}$ contains the elements

$$
V_{i j}=\mu_{\mathrm{sp}}^{2} / R_{i j}^{3}
$$

and $I$ is the $N \times N$ unit matrix.

In a first step, we can diagonalize the matrix $\underline{\underline{V}}$ :

$$
\underline{\underline{V}} \vec{a}^{(l)}=E^{(l)} \vec{a}^{(l)},
$$

where $l$ labels the $N$ eigenvectors. Then Eq. (A1) can be written as

$$
\begin{aligned}
H(B)\left[\underline{I} \otimes \vec{a}^{(l)}\right] & =\underline{\epsilon(B)} \otimes \vec{a}^{(l)}+E^{(l)} \underline{M} \otimes \vec{a}^{(l)} \\
& =\left[\underline{\epsilon(B)}+E^{(l)} \underline{M]} \otimes \vec{a}^{(l)} .\right.
\end{aligned}
$$

In the next step, we diagonalize for each $l$ the $3 \times 3$ matrices $\underline{\epsilon(B)}+E^{(l)} \underline{M}$ :

$$
\left[\underline{\epsilon(B)}+E^{(l)} \underline{M}\right] \vec{b}^{(l, \kappa)}(B)=E^{(l, \kappa)}(B) \vec{b}^{(l, \kappa)}(B) .
$$

Here $\kappa$ labels the three eigenvectors of each $\kappa$-block. With this we finally can write

$$
H(B)\left[\vec{b}^{(l, \kappa)}(B) \otimes \vec{a}^{(l)}\right]=E^{(l, \kappa)}(B)\left[\vec{b}^{(l, \kappa)}(B) \otimes \vec{a}^{(l)}\right] .
$$

We can combine the two labels $l$ and $\kappa$ into a single label $\ell$ and define as eigenfunctions

$$
\vec{c}^{(\ell)}=\vec{b}^{(l, \kappa)} \otimes \vec{a}^{(l)} .
$$

From this we can make the identification

$$
c_{j, m}^{(\ell)}=b_{m}^{(l, \kappa)} a_{j}^{(l)} .
$$

Since we are interested in the populations on each site [see Eq. (15)], we find

$$
P_{j}^{(l, \kappa)}=\left(\sum_{m}\left|b_{m}^{(l, \kappa)}\right|^{2}\right)\left|a_{j}^{(l)}\right|^{2} .
$$

[1] H. van Amerongen, L. Valkunas, and R. van Grondelle, Photosynthetic Excitons (World Scientific, Singapore, 2000).

[2] J-Aggregates, edited by T. Kobayashi (World Scientific, Singapore, 1996).

[3] S. K. Saikin, A. Eisfeld, S. Valleau, and A. Aspuru-Guzik, Nanophotonics 2, 21 (2013).

[4] S. A. Crooker, J. A. Hollingsworth, S. Tretiak, and V. I. Klimov, Phys. Rev. Lett. 89, 186802 (2002).

[5] B. D. Gerardot, S. Strauf, M. J. A. de Dood, A. M. Bychkov, A. Badolato, K. Hennessy, E. L. Hu, D. Bouwmeester, and P. M. Petroff, Phys. Rev. Lett. 95, 137403 (2005).

[6] M. Quinten, A. Leitner, J. R. Krenn, and F. R. Aussenegg, Opt. Lett. 23, 1331 (1998).

[7] W. R. Anderson, J. R. Veale, and T. F. Gallagher, Phys. Rev. Lett. 80, 249 (1998).

[8] T. Gallagher, Rydberg Atoms (Cambridge University Press, Cambridge, 2005).

[9] F. Robicheaux, J. V. Hernández, T. Topçu, and L. D. Noordam, Phys. Rev. A 70, 042703 (2004).
Since $\left(\sum_{m}\left|b_{m}^{(l, \kappa)}\right|^{2}\right)=1$, we can finally write

$$
P_{j}^{(l, \kappa)}=\left|a_{j}^{(l)}\right|^{2} .
$$

From this one sees that the populations are independent of the magnetic field strength and direction. They are given by the $m$-level independent Hamiltonian $\underline{\underline{V}}$, which corresponds to isotropic interaction.

Alternative considerations using $B=0$ : With $B=0$ it is convenient to choose the quantization axis such that all coupling elements $\left(M_{i, j}^{0, \pm 1}, M_{i, j}^{ \pm 1,0}\right.$, and $\left.M_{i, j}^{ \pm 1, \mp 1}\right)$ vanish. This happens when the quantization axis is parallel to the chain $(\theta=0)$. That means that the $m=-1,0$, and +1 states are uncoupled and the Hamiltonian has a block-diagonal form, where each block belongs to a specific $m$-state. Each block can be diagonalized independently. From the definition of $M_{i, j}^{m_{i}, m_{j}}$ one sees that the three subblocks have the form

$$
H^{(m)}=\sum_{i, j}\left[\epsilon \delta_{i j}+\frac{\mu_{\mathrm{sp}}^{2}}{R^{3}} \frac{1}{|i-j|^{3}} M^{(m)}\right]|i, m\rangle\langle j, m|
$$

with $M^{(0)}=1 / 3$ and $M^{( \pm 1)}=-1 / 6$, and where $R$ is the lattice spacing. Since $M^{(m)}$ is independent of the atomic position $i$ and $j$, it simply scales the interaction strength. Therefore, one has identical eigenstates for each sub-Hamiltonian.

\section{APPENDIX B: PTM ESTIMATE FOR THE 1D CHAIN}

The result (A13) can be used to analytically estimate the extent of the delocalization in the case of a 1D linear chain. To obtain a simple analytical expression, we take only the interaction between nearest neighbor s into account. Then, the squares of the eigenfunction coefficients are given by $\left|a_{j}^{(l)}\right|^{2}=$ $2 /(N+1) \sin ^{2}(\pi j \ell /(N+1))$. One sees that roughly half of them are larger than the threshold $P_{\text {thresh }}=1 / N$. Therefore, we expect the eigenstates to have a PTM value of approximately $N / 2$. As we discuss in Sec. V A, this estimated value agrees very well with the numerical result presented in Fig. 4.

[10] W. Li, J. P. Tanner, Y. Jamil, and F. T. Gallagher, Eur. Phys. J. D 40, 27 (2006).

[11] H. Ditlbacher, J. R. Krenn, B. Lamprecht, A. Leitner, and F. R. Aussenegg, Opt. Lett. 25, 563 (1999).

[12] D. Barredo, H. Labuhn, S. Ravets, T. Lahaye, A. Browaeys, and C. S. Adams, Phys. Rev. Lett. 114, 113002 (2015).

[13] H. Fidder, J. Knoester, and D. A. Wiersma, J. Chem. Phys. 95, 7880 (1991).

[14] M. V. Mostovoy, M. T. Figge, and J. Knoester, Phys. Rev. B 57, 2861 (1998).

[15] A. Eisfeld, G. Schulz, and J. S. Briggs, J. Lumin. 131, 2555 (2011).

[16] L. Bruder, A. Eisfeld, U. Bangert, M. Binz, M. Jakob, D. Uhl, M. Schulz-Weiling, E. R. Grant, and F. Stienkemeier, Phys. Chem. Chem. Phys. 21, 2276 (2019).

[17] A. Goetschy and S. E. Skipetrov, Phys. Rev. E 84, 011150 (2011).

[18] A. Goetschy and S. E. Skipetrov, arXiv:1303.2880.

[19] V. M. Akulin, Dynamics of Complex Quantum Systems (Springer, Dordrecht, 2014). 
[20] T. Scholak, T. Wellens, and A. Buchleitner, Phys. Rev. A 90, 063415 (2014).

[21] C. Didraga and J. Knoester, J. Chem. Phys. 121, 10687 (2004).

[22] M. V. Mostovoy and J. Knoester, J. Phys. Chem. B 104, 12355 (2000).

[23] J. M. Moix, Y. Zhao, and J. Cao, Phys. Rev. B 85, 115412 (2012).

[24] I. Barvík, C. Warns, and P. Reineker, J. Lumin. 119-120, 504 (2006).

[25] J.-L. Brédas, E. H. Sargent, and G. D. Scholes, Nat. Mater. 16, 35 (2017).

[26] J. M. Gottfried, Surf. Sci. Rep. 70, 259 (2015).

[27] S. M. Vlaming and A. Eisfeld, J. Phys. D 47, 305301 (2014).

[28] Y. Zhang, Y. Luo, Y. Zhang, Y.-J. Yu, Y.-M. Kuang, L. Zhang, Q.-S. Meng, Y. Luo, J.-L. Yang, Z.-C. Dong, and J. G. Hou, Nature (London) 531, 623 (2016).

[29] Y. Luo, G. Chen, Y. Zhang, L. Zhang, Y. Yu, F. Kong, X. Tian, Y. Zhang, C. Shan, Y. Luo, J. Yang, V. Sandoghdar, Z. Dong, and J. G. Hou, Phys. Rev. Lett. 122, 233901 (2019).

[30] J. Yuen-Zhou, S. K. Saikin, N. Y. Yao, and A. Aspuru-Guzik, Nat. Mater. 13, 1026 (2014).

[31] A. Browaeys, D. Barredo, and T. Lahaye, J. Phys. B 49, 152001 (2016).

[32] S. Möbius, S. Wüster, C. Ates, A. Eisfeld, and J.-M. Rost, J. Phys. B 44, 184011 (2011).

[33] A. Paris-Mandoki, H. Gorniaczyk, C. Tresp, I. Mirgorodskiy, and S. Hofferberth, J. Phys. B 49, 164001 (2016).
[34] G. Günter, H. Schempp, M. Robert-de Saint-Vincent, V. Gavryusev, S. Helmrich, C. S. Hofmann, S. Whitlock, and M. Weidemüller, Science 342, 954 (2013).

[35] G. Abumwis, M. T. Eiles, and A. Eisfeld, J. Phys. B 53, 124003 (2020).

[36] G. Abumwis, M. T. Eiles, and A. Eisfeld, Phys. Rev. Lett. 124, 193401 (2020).

[37] A. Browaeys and T. Lahaye, Nat. Phys. 16, 132 (2020).

[38] D. Barredo, V. Lienhard, S. De Leseleuc, T. Lahaye, and A. Browaeys, Nature (London) 561, 79 (2018).

[39] S. Hollerith, J. Zeiher, J. Rui, A. Rubio-Abadal, V. Walther, T. Pohl, D. M. Stamper-Kurn, I. Bloch, and C. Gross, Science 364, 664 (2019).

[40] D. Ohl de Mello, D. Schäffner, J. Werkmann, T. Preuschoff, L. Kohfahl, M. Schlosser, and G. Birkl, Phys. Rev. Lett. 122, 203601 (2019).

[41] I. S. Madjarov, J. P. Covey, A. L. Shaw, J. Choi, A. Kale, A. Cooper, H. Pichler, V. Schkolnik, J. R. Williams, and M. Endres, Nat. Phys. 16, 857 (2020).

[42] D. Peter, N. Y. Yao, N. Lang, S. D. Huber, M. D. Lukin, and H. P. Büchler, Phys. Rev. A 91, 053617 (2015).

[43] C. Lippe, T. Klas, J. Bender, P. Mischke, T. Niederprüm, and H. Ott, arXiv:2012.12739.

[44] A. Eisfeld, C. Marquardt, A. Paulheim, and M. Sokolowski, Phys. Rev. Lett. 119, 097402 (2017).

[45] M. Müller, A. Paulheim, A. Eisfeld, and M. Sokolowski, J. Chem. Phys. 139, 044302 (2013).

[46] V. Lienhard, P. Scholl, S. Weber, D. Barredo, S. de Léséleuc, R. Bai, N. Lang, M. Fleischhauer, H. P. Büchler, T. Lahaye et al., Phys. Rev. X 10, 021031 (2020). 